\title{
Laser assisted decay spectroscopy at the CRIS beam line at ISOLDE
}

\author{
K. M. Lynch • T. E. Cocolios • M. M. Rajabali \\ on behalf of the CRIS collaboration
}

Published online: 7 February 2013

(C) Springer Science+Business Media Dordrecht 2013

\begin{abstract}
The new collinear resonant ionization spectroscopy (CRIS) experiment at ISOLDE, CERn uses laser radiation to stepwise excite and ionize an atomic beam for the purpose of ultra-sensitive detection of rare isotopes and hyperfine structure measurements. The technique also offers the ability to purify an ion beam that is contaminated with radioactive isobars, including the ground state of an isotope from its isomer. A new program using the CRIs technique to select only nuclear isomeric states for decay spectroscopy commenced last year. The isomeric ion beam is selected using a resonance within its hyperfine structure and subsequently deflected to a decay spectroscopy station. This consists of a rotating wheel implantation system for alpha and beta decay spectroscopy, and up to three high purity germanium detectors for gamma-ray detection. This paper gives an introduction to the CRIs technique, the current status of the laser assisted decay spectroscopy set-up and recent results from the experiment in November 2011.
\end{abstract}

Keywords CRIS · ISOLDE • Laser spectroscopy • Decay spectroscopy

Proceedings of the 6th international conference on Laser Probing (LAP 2012),

Paris, France, 4-8 June 2012.

K. M. Lynch (西)

School of Physics and Astronomy,

The University of Manchester, Manchester, M13 9PL, UK

e-mail: kara.marie.lynch@cern.ch

K. M. Lynch · T. E. Cocolios

Physics Department, CERN, 1211 Geneva 23, Switzerland

M. M. Rajabali

Instituut voor Kern- en Stralingsfysica,

KU Leuven, 3001 Leuven, Belgium

Present Address:

M. M. Rajabali

TRIUMF, Vancouver, BC, V6T 2A3, Canada 


\section{Introduction}

The CRIS beam line combines two complementary spectroscopic techniques to provide a wealth of information on the nuclear species under investigation [1-3]. Laser spectroscopy allows nuclear observables to be extracted with nuclear model independence: the nuclear spin, moments and the change in the mean-square charge radii $\left(\delta\left\langle r^{2}\right\rangle\right)$ between isotopes [4]. From the complementary decay spectroscopy, level scheme information on the daughter nucleus can be obtained. The CRIS technique provides a combination of high detection efficiency, high resolution and ultra-low background, allowing measurements to be performed on isotopes with yields down to 1 atom per second.

\section{Collinear resonant ionization spectroscopy}

Laser radiation is used to resonantly excite and ionize an atomic beam, probing the atomic transitions of an isotope. These transitions are used to extract nuclear observables, such as magnetic dipole and electric quadrupole moments, as well as $\delta\left\langle r^{2}\right\rangle$ between isotopes. A sequence of two transitions at selected frequencies are required to bring the electron across the ionization potential. When the laser frequencies are on resonance with the hyperfine component of the optical transition, the isotope is ionized. By using short laser pulses, loses associated with optical pumping can be avoided. This process of resonant ionization [5-7] selects the isotope of interest. The maximum selectivity $S$, of an isotope from an isomer or another isotope is given by the equation

$$
S=\left(\frac{\Delta \omega_{A B}}{\Gamma}\right)^{2}=\prod S_{n},
$$

where $\Delta \omega_{A B}$ is the separation between the respective hyperfine components and $\Gamma$ is the width of those transitions, Fig. 1a. Additional selectivity can be gained due to the kinematic shift between different masses of the isotopes caused by the collinear geometry. The total selectivity of a resonant ionization process is given by the product of the individual selectivities, thus the higher the number of atomic excitation steps, the greater the selectivity of the nuclear state, as illustrated in Fig. 1b. Indeed, the selectivity of resonant ionization can be compared to the mass resolution of a state in mass spectrometry. The sensitivity of the technique comes from the detection of resonant ions (with the possibility of $100 \%$ detection efficiency), efficient ionization (between 10-30 \%) and almost background free detection.

Due to the high degree of selectivity and efficiency of the resonant ionization process, the CRIS technique can be utilized as a purification method [8]. The different nuclear observables (spin, moments, $\delta\left\langle r^{2}\right\rangle$ ) of isotopes and isomers produce different hyperfine structures, resulting in the lasers only ionizing the nuclear state of interest while on resonance with a characteristic transition. This results in the ability to perform decay spectroscopy on pure isomeric states with a suppression of the ground state by a factor of at least $10^{4}$ per resonant transition. 


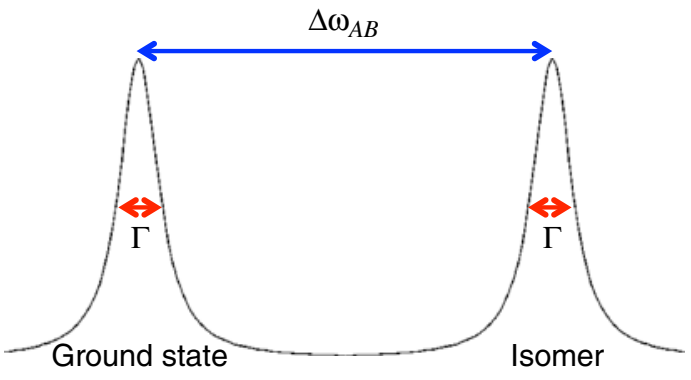

(a)

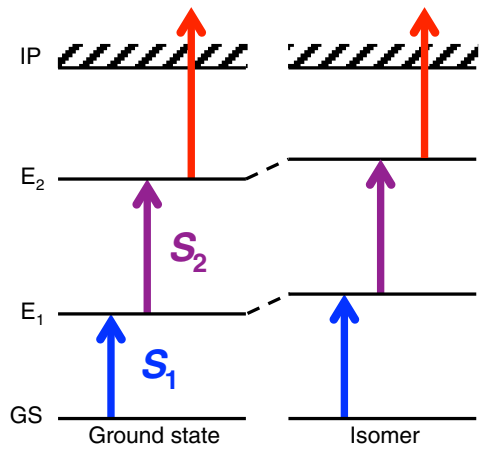

(b)

Fig. 1 a The difference in frequency $\Delta \omega_{A B}$ between two absorption lines, due to the isomer shift. b The number of resonant transitions increases the selectivity of the process

\section{Laser assisted decay spectroscopy}

Using the technique of in-source laser spectroscopy [9], the identification and use of isomeric beams has already been achieved, for example in the copper isotopes ${ }^{68,70} \mathrm{Cu}[10,11]$. However, a large isobaric contamination in the form of surface ionized gallium was present, along with a ground state contamination due to the Doppler broadening of the hyperfine resonances of each state. Collinear laser spectroscopy [12] successfully identified the long-lived isomer in ${ }^{80} \mathrm{Ga}$ that was not observed by mass measurements [13]. Higher resolution can be provided by gamma ray spectroscopy, but distinctions between ground and isomeric states that both betadecay cannot be made. With collinear laser spectroscopy, isomeric states differing by only $1 \mathrm{keV}$ in energy can be resolved through their hyperfine structure, but with high-resolution gamma spectroscopy they cannot be seen as independent levels, for example in ${ }^{73} \mathrm{Ga}$ [14]. The alpha decay of the isomeric states of ${ }^{202} \mathrm{Fr}$ and ${ }^{204} \mathrm{Fr}$ could not initially be differentiated with decay spectroscopy [15]. Later measurements gave tentative spin-parity assignments to these low-lying isomers, based on feeding patterns in $\beta^{+} / \mathrm{EC}$ decays and on systematics with the neighbouring isotopes and isotones.

As a proof of principle for the CRIS technique $[8,16]$, the first case to be investigated was the low lying isomerism in ${ }^{204} \mathrm{Fr}$ [17]. Here, the ground and isomeric states are within $41 \mathrm{keV}$ of each other [18-20]. This region of the nuclear chart has already received considerable attention, with experimental campaigns into $\beta$-delayed fission of ${ }^{200,202} \mathrm{Fr}$ already undertaken [21]. Radioactive decay spectra from the ground state, first and second isomeric states in ${ }^{204} \mathrm{Fr}$ have been difficult to unravel; the emitted alpha particles have very similar energies and half lives. By exploiting the different hyperfine structures and locking the laser onto resonance with a characteristic transition, differentiation between the three states becomes possible. Thus the radioactive decays of pure beams can be measured, allowing the energies of the alpha particles and gamma rays emitted, and the lifetime of the states, to be unambiguously determined. 


\section{The CRIS beam line at ISOLDE}

Francium isotopes are produced by impinging $1.4 \mathrm{GeV}$ protons on a thick target $\left(\mathrm{UC}_{x}\right.$ or $\left.\mathrm{ThC}_{x}\right)$. These radioactive products are then surface ionized, accelerated to $30 \mathrm{keV}$ and mass separated with the high resolution separator (Hrs) to select the francium isotope of interest [22]. The ions are then bunched using the radiofrequency quadrupole Iscool [23]. The bunching of the ion beam is essential for removing the duty cycle losses due to the pulsed lasers. Alternative stable beam can be produced from the CRIS off line ion source, which has the ability to produce caesium or rubidium ions. These beams allow for the testing of ionization schemes and the optimization of transmission through the CRIS beam line. The ion beam is passed through an alkali vapour charge exchange cell (CEC), which neutralizes the bunch in preparation for resonant ionization. Here, a Doppler tuning voltage is applied to the ions entering the $\mathrm{CEC}$, adapting the Doppler shift of the locked frequency of the lasers relative to the interacting atomic bunch.

In the interaction region, the atom bunch is collinearly overlapped with two laser beams. The collinear geometry of the set-up provides a reduction in thermal Doppler broadening by a factor of $\approx 10^{3}$, increasing the selectivity of the state of interest. This is because the product of the velocity and velocity spread remains constant under acceleration. This decreases the Doppler broadened line width of the hyperfine transition to below its natural width, which leads to a greater degree of selectivity between the ground and isomeric states of the isotope, and thus higher resolution and sensitivity to the nuclear observables. The first transition is from the $7^{2} \mathrm{~S}_{1 / 2}$ electronic orbital to the $8^{2} \mathrm{P}_{3 / 2}$ state with $422 \mathrm{~nm}$ light. The second (non-resonant) transition is from the $8^{2} \mathrm{P}_{3 / 2}$ state into the continuum using $1064 \mathrm{~nm}$ light. A new injectionseeded Ti:Sa laser system was used to produce these two laser beams [24]. The atoms were ionized when the laser light is on resonance with a hyperfine transition and deflected to the decay spectroscopy station (Dss). Here, they can be detected with an MCP, for hyperfine structure measurements, or implanted into a carbon foil for decay measurements.

The Dss consists of a rotating wheel implantation system, based on the design from KU Leuven [25, 26], and shown in Fig. 2 [27]. Four Canberra silicon PIPS detectors are installed in the Dss for alpha and beta decay detection. Additionally, three high-purity germanium detectors can be placed around the implantation site for gamma-ray detection. A Canberra high purity germanium SEGe detector and an Eurisys thin beryllium window x-ray detector were used during the experiment. Pure beams of the desired isotope or isomer can be deflected to the decay station, where alpha and gamma decays are detected. The data was recorded event by event using a fully digital data acquisition system. Alpha-gamma coincidences were then reconstructed off-line to identify a radioactive decay, and reduce the background associated with the dumped beam or the environment.

\section{Recent results from spectroscopy on francium isotopes at ISOLDE}

Laser assisted decay spectroscopy was successfully performed on ${ }^{207} \mathrm{Fr}$, with an increase in the number of alpha particles detected when the laser frequency was on resonance with the hyperfine transitions in the francium isotope by a factor of 


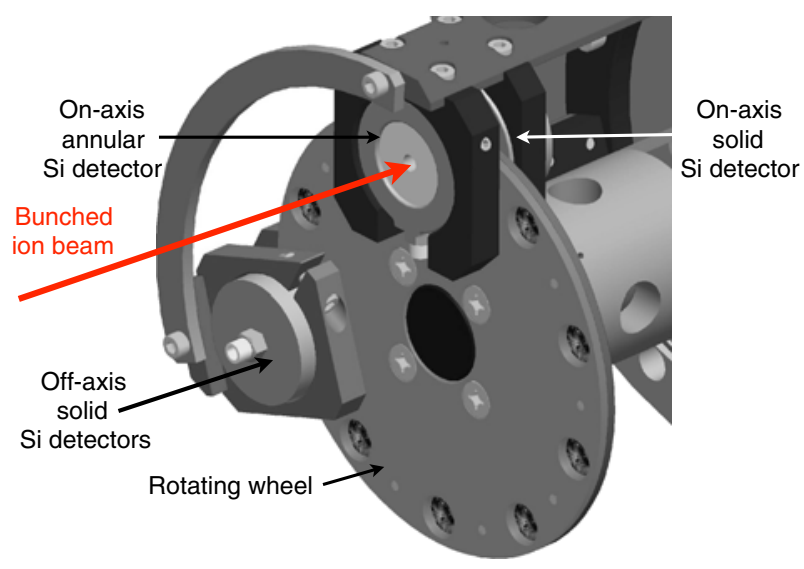

Fig. 2 Technical drawing of the Dss wheel and silicon detectors. The wheel holds ten thin carbon foils, with a thickness of $800 \mathrm{~nm}$ into which the ionized bunch is implanted. Two Canberra PIPS silicon detectors for alpha decay detection are situated on either side of the implanted carbon foil. One solid detector (Series A, $300 \mu \mathrm{m}$ thick) sits behind the carbon foil, and an annular detector (Series AN, $300 \mu \mathrm{m}$ thick) is placed in front. The ion beam is directed through the $4 \mathrm{~mm}$ aperture of the annular detector, allowing for backwards recoil particle detection. In addition to the on-axis detectors, two solid PIPS silicon detectors are placed off-axis. This allows for measurements on longer lived decay products to be performed by rotating the implanted foil from the on-axis to off-axis detector position

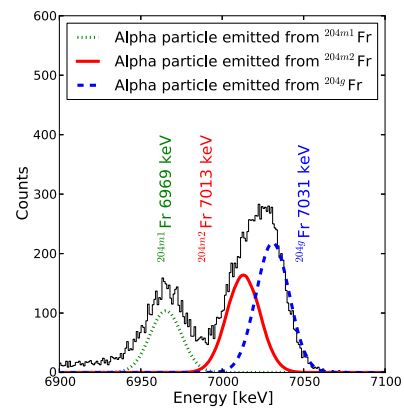

(a)

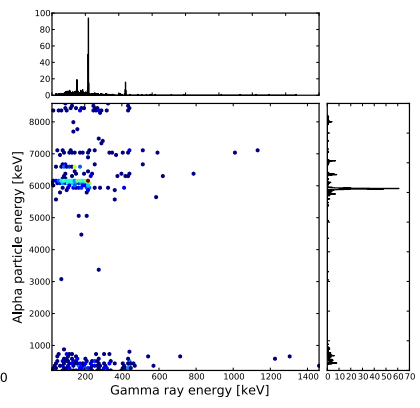

(b)

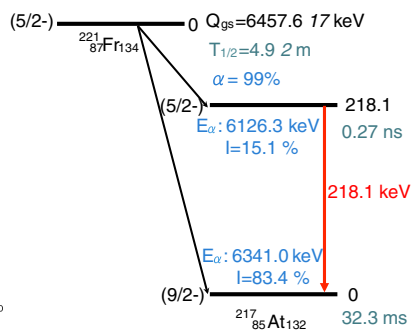

(c)

Fig. 3 a Simulated single lines are the expected alpha-particle energy spectra for the ground state (dashed), first isomeric state (dotted) and second isomeric state (solid) for ${ }^{204} \mathrm{Fr}$ when the laser is on resonance with a characteristic hyperfine transition. b Gamma-ray energy spectrum (top), alpha-particle energy spectrum (right) and alpha-gamma energy matrix showing the alpha-gamma coincidence for the radioactive decay of ${ }^{221} \mathrm{Fr}$. $\mathbf{c}$ The radioactive decay of ${ }^{221} \mathrm{Fr}$

$66 \pm 11$. The ratio of detected alpha particles when the laser was on resonance to when the laser was blocked, can be increased in several ways. Any increase in the overall efficiency of the experiment, e.g. laser ionization efficiency, will increase the number of alpha-particle decays detected. Additionally, improving the vacuum in the interaction region will decrease the amount of non-resonant ionizations due to collisional excitations. Decay spectroscopy was performed on ${ }^{204} \mathrm{Fr}$, with the alpha particles emitted from ${ }^{204 g, m 1, m 2} \mathrm{Fr}$ shown in Fig. 3a. This figure illustrates the necessity of being able to distinguish the individual states of the ${ }^{204} \mathrm{Fr}$ isotopes. Here, 
the alpha-particle energies of ${ }^{204 g} \mathrm{Fr}$ and ${ }^{204 m 2} \mathrm{Fr}$ cannot be resolved, leading to large uncertainties in the energies of the alpha particles and half lives of the states. By using the characteristic hyperfine structure of each state, a pure ground state or isomeric beam can be studied. Thus, by tuning the lasers onto resonance with a hyperfine transition particular to ${ }^{204 m 2} \mathrm{Fr}$, the solid line spectrum shown in Fig. 3a would be obtained. Furthermore, the dashed line spectrum would be observed if the laser was tuned onto resonance with a transition in ${ }^{204 g} \mathrm{Fr}$. Alpha-gamma coincidences in ${ }^{221} \mathrm{Fr}$ were observed, shown in Fig. 3b. These result from the $6126.3 \mathrm{keV}$ alpha-particle decay of ${ }^{221} \mathrm{Fr}$ to ${ }^{217} \mathrm{At} *$ followed by prompt gamma-ray emission $(218.1 \mathrm{keV})$ to its ground state ${ }^{217}$ At, see Fig. 3c. This demonstrates the ability of the Dss setup to obtain alpha-gamma coincidences, advantageous for the identification of radioactive decay schemes.

\section{Summary}

The selectivity of collinear resonant ionization spectroscopy can be utilised to perform decay spectroscopy on pure isomeric beams. An experimental campaign is underway at the CRIS beam line to use complementary laser and decay spectroscopy techniques to study pure ground state and isomeric states of francium. This includes the detailed study of the two isomers of ${ }^{202} \mathrm{Fr}$ for $\beta$-delayed fission [21], along with other francium nuclei in the isotopic chain, ${ }^{201-206,218,219}$ Fr. Laser assisted decay spectroscopy was performed on ${ }^{207} \mathrm{Fr}$ in November 2011, displaying an increase in alpha particles detected when the lasers were on resonance by a factor of $66 \pm 11$.

Acknowledgements We thank the the ISOLDE team for providing excellent beams, the KU Leuven technical staff for their support and assistance, and the GSI target laboratory for manufacturing the carbon foils. This work is supported by the U.K. Science and Technology Facilities Council, by FWO-Vlaanderen (Belgium), by GOA/2004/03 (BOF-KULeuven), by the IUAP -Belgian State Belgian Science Policy (BriX network P6/23), and by the European Commission within the Seventh Framework Programme through ENSAR (Contract No. 262010).

\section{References}

1. Flanagan, K.T., et al.: AIP Conf. Proc. 1377, 38 (2011)

2. Procter, T.J., et al.: J. Phys.: Conf. Ser. 381, 012070 (2012)

3. Procter, T.J., et al.: LAP2012 Proceedings (2012)

4. Cheal, B., Flanagan, K.T.: J. Phys. G 37(11), 113101 (2010)

5. Hurst, G.S., et al.: Rev. Mod. Phys. 51(4), 767 (1979)

6. Billowes, J., Campbell, P.: J. Phys. G: Nucl. Part. Phys. 21, 707 (1995)

7. Fedosseev, V.N., Kudryavtsev, Yu., Mishin, V.I.: Phys. Scr. 85, 058104 (2012)

8. Letokhov, V.: Opt. Commun. 7, 59 (1973)

9. Weissman, L., et al.: Phys. Rev. C 65, 024315 (2002)

10. Van Roosbroeck, J., et al.: Phys. Rev. Lett. 92, 112501 (2004)

11. Stefanescu, I., et al.: Phys. Rev. Lett. 98, 122701 (2007)

12. Cheal, B., et al.: Phys. Rev. C 82, 051302 (2010)

13. Hakala, J., et al.: Phys. Rev. Lett. 101, 052502 (2008)

14. Cheal, B., et al.: Phys. Rev. Lett. 104, 252502 (2010)

15. Hornshoj, P., Hansen, P., Jonson, B.: Nucl. Phys. A 230(3), 380 (1974)

16. Schulz, Ch., et al.: J. Phys. B At. Mol. Opt. Phys. 24, 4831 (1991)

17. Lynch, K.M., et al.: J. Phys.: Conf. Ser. 381, 012128 (2012)

18. Huyse, M., et al.: Phys. Rev. C 46, 1209 (1992) 
19. Chiara, C., Kondev, F.: Nucl. Data Sheets 111(1), 141 (2010)

20. Uusitalo, J., et al.: Phys. Rev. C 71, 024306 (2005)

21. Andreyev, A.N., et al.: CERN-INTC-2008-001, INTC-P-235. CERN Geneva (2008)

22. Jonson, B., Richter, A.: Hyperfine Interact. 129, 1 (2000)

23. Mané, E., et al.: Eur. Phys. J. A 42, 503 (2009)

24. Hori, M., Dax, A.: Opt. Lett. 34(8), 1273 (2009)

25. Dendooven, P.: Ph.D. thesis, Katholieke Universiteit Leuven (1992)

26. Andreyev, A.N., et al.: Phys. Rev. Lett. 105, 252502 (2010)

27. Rajabali, M.M., et al.: Nucl. Instrum. Methods Phys. Res. A 707, 35 (2013). doi:10.1016/j.nima. 2012.12.090i 